\title{
Magnetic field-induced self-assembly of chemically modified graphene oxide on cellulose fabrics for the fabrication of flexible conductive devices
}

\author{
Huanxia Zhang $\mathbb{D} \cdot$ Wen Wu $\cdot$ Jie Zhou $\cdot$ Xinchao Zhang $\cdot$ Tantan Zhu \\ Mingqiong Tong
}

Received: 12 June 2020/ Accepted: 16 December 2020/Published online: 6 January 2021

(C) The Author(s) 2021

\begin{abstract}
In this present study, we have successfully fabricated the cellulose fabric with excellent electrical conductivity by depositing the graphene oxide grafted with the modified ferroferric oxide (GOF) upon fabric substrate via layer-by-layer magnetic-field-induced self-assembly apporoach and followed by chemical reduction. The results indicated that the morphologies of graphene oxide nanosheets for three-layer deposited fabrics could form the highly oriented wrinkled structures, which resulted from the synergistic interactions of magnetic induction force on magnetic doublet, hydrogen bonds and van der Waals. The volume resistivity of the three-layer deposited fabric could reach to $64.8 \Omega \mathrm{cm}$ compared with that of pure RGO-coated viscose fabrics $(137.94 \Omega \mathrm{cm})$ in the previous work, which could be favorable for improving the electrical conductivity and decreasing the graphene oxide consumption. Furthermore, the threelayer deposited fabric possessed excellent washing durability even after twelve times water laundering.
\end{abstract}

H. Zhang $(\bowtie) \cdot$ W. Wu $~ J$ J. Zhou $\cdot$ X. Zhang $\cdot$ T. Zhu College of Material and Textile Engineering, Jiaxing University, Jiaxing 314001, China

e-mail: zhanghuanxia818@163.com

M. Tong

Shandong Provincial Engineering Laboratory of Novel Pharmaceutical Excipients, Sustained and Controlled Release Preparations, College of Medicine and Nursing, Dezhou University, Decheng District, Dezhou 253023, China
Our results suggested that the flexible GOF-coated fabric had great potential in conductive devices for wearable electronics, strain sensors, smart actuators and bioelectrodes and so on.

Keywords Graphene oxide - Ferroferric oxide . Alternating ordered structure $\cdot$ Magnetic field induction - Electrical conductivity

\section{Introduction}

High-performance smart textiles with electrical conductivity are an integral part of modern industry and life, being used in many applications such as health, sport, automotive, aerospace and military ( $\mathrm{Gu}$ et al. 2010; Grancarić et al. 2017; Heidari et al. 2019; Zhao et al. 2019). Recently, research on graphene was motivated by its fascinating mechanical and electrical properties. Graphene oxide (GO), the oxygenated form of a monolayer graphene sheet, that can be conveniently dissolved in an aqueous solution offer the potential for the rational design of graphene-based functional fabrics (Kim, Tae Young et al. 2011, Chen et al. 2012; Song et al. 2013). For example, Gao et al. designed and fabricated ultrastrong graphene fibers with giant graphene oxide sheets that had the excellent electrical conductivity and possessed a record tensile strength (up to $0.5 \mathrm{GPa}$ ) among neat graphene materials (Xu et al. 2013). Zhu and co-workers have 
developed the non-ignitable electrically conductive polyester/cotton fabric modified by reduced graphene oxide and phosphate flame retardant, which has a low electrical surface resistivity $(0.54 \mathrm{~K} \Omega / \mathrm{sq})$ (Zhao, Wang et al. 2019). Comparing with other substrate materials, the introduction of fabrics could provide higher surface area for the deposition of other materials (such as graphene nanosheets) by using dip coating method and increase number of electroactive areas for access electrolyte ions, which helped to improve the performance of electrical conductivity (Tao et al. 2010; Bao et al. 2011; Ramadoss et al. 2015). Tian et al. prepared soft bimorph actuator, stretchable electrical conductors, self-sensing and self-cooling integrated smart sportswear and versatile fabric by depositing functionalized graphene via coating or layer-by-layer electrostatic self-assembly method on the surface of fabric or fibers, which exhibited the excellent electronic conductive and selfsensing properties (Zhao et al. 2020a, b; Sun et al. 2019; Hu et al. 2020; Tian et al. 2016).

These excellent properties of graphene-based electronic fabrics were determined by the microstructures of graphene sheets, such as ripples, kinks and folds (Gómez-Navarro et al. 2008; Shenoy et al. 2008, 2010; Huang et al. 2009). However, for the assembly of graphene from suspended two-dimensional sheets to macroscopic structures, a critical issue encountered in achieving graphene fabrics with both excellent mechanical properties and high electrical conductivity is the graphene nanosheets agglomeration. Therefore, the microstructure manipulation of these products would play an important role to improve their performance and application fields. Huang et al. prepared the thin films of assembled crumpled graphene balls by changing two-dimensional graphene sheets into three-dimensional crumpled balls used as the strong light absorbers to prevent the re-stacking of GO sheets, which revealed that the average absorption can reach up to $97.4 \%$ in the visible and near-infrared regions. Particularly, the submicron sized reduced graphene oxide particles with a paper ball-like morphology was the components of the crumpled graphene balls, which was aggregation-resistant and will not stack to form highly reflective microstructures, even after densification (Hao et al. 2018). Fu et al. synthesized a two-dimensional superordered structure via self-assembly of high-quality graphene single crystals, which revealed that the electrostatic interactions between the adjacent crystals facilitate the ultimate precise orientation with each other (Zeng et al. 2016).

The agglomeration problem of graphene also can be effectively solved by an external field. It is well known that the applied magnetic field can significantly influence the movement of magnetic nanoparticles. It is, therefore, significant to study the arrangement and self-assembly behavior of magnetic nanoparticles deposited on the graphene fabrics under an external magnetic field. $\mathrm{Li}$ et al. fabricated successfully composite material with graphene shell by using a novel internal magnetic field enhanced polysulfide trapping mechanism, which provides an effective method for controlling the spatial and temporal stacking structure of grapheme (Gao et al. 2018). The layer-by-layer assembly method has been widely used for finishing fibers or fabric with graphene along with the advantages of a controlled assembly structure. However, little attention has been paid to the layer-bylayer self-assembly behavior of a magnetic field on the graphene fabrics with magnetic nanoparticles and the corresponding mechanisms. In our work, we designed a layer-by-layer magnetic-field-induced self-assembly of graphene oxide sheets grafted with ferroferric oxide (GOF) and followed by chemical reduction to fabricate the cellulose fabric with a control deformation of GO sheets. The morphology and nanostructure of different cellulose fabrics were characterized and the alignment mechanism of GO nanosheets was also analyzed. Moreover, the flexible electrical and thermal conductivity properties of the different coated-fabrics were measured and the relationship between the different structure of the assembly layer-deposited fabrics and their properties was further investigated. Our results suggested that the flexible GOF-coated fabric via the magnetic-field induction would contribute to the future production of wearable electronics.

\section{Experimental sections}

\section{Materials}

Graphite was purchased from Sigma-Aldrich. In addition, Iron(III) chloride hexahydrate, Iron(II) chloride tetrahydrate, Silane coupling agent KH-560, sodium hydroxide, hydrochloric acid, methanol, 
toluene, HI acid, sulfuric acid, hydrogen peroxide, 1-ethyl-3-(3-dimethylaminopropyl) carbodiimide hydrochloride (EDC), sodium nitrate and potassium permanganate were supplied by Sinopharm chemical reagent Co., Ltd. Cellulose fabric was purchased from Suzhou Shuishan Industrial Co., Ltd, Jiangsu, China and was viscose woven structure with $141 \mathrm{~g} / \mathrm{m}^{2}$.

Preparation of $\mathrm{Fe}_{3} \mathrm{O}_{4}$ and amino-modified $\mathrm{Fe}_{3} \mathrm{O}_{4}$ nanoparticles

The solid sample of $\mathrm{NaOH}(5.0 \mathrm{~g})$ was added into the deionized water $(250 \mathrm{~mL})$, heated to $80{ }^{\circ} \mathrm{C}$. The samples, including $\mathrm{FeCl}_{3} \cdot 6 \mathrm{H}_{2} \mathrm{O}(6.75 \mathrm{~g}), \mathrm{FeCl}_{2} \cdot 4 \mathrm{H}_{2} \mathrm{O}$ $(2.48 \mathrm{~g})$, the deionized water $(25 \mathrm{~mL})$ and $\mathrm{HCl}$ $(0.83 \mathrm{~mL})$ were mixed respectively into the heated $\mathrm{NaOH}$ solution and loaded into the three-necked flask. The reaction was continued for $1 \mathrm{~h}$ under the protection of nitrogen (Han et al. 2007; Kim et al. 2011). Then, the solution was vacuum-filtered, resulting in black slurry. The obtained slurry was washed with water and methyl alcohol many times until the $\mathrm{pH}$ of the slurry was 7. The magnetic $\mathrm{Fe}_{3} \mathrm{O}_{4}$ nanoparticle was finally obtained. To endow $\mathrm{Fe}_{3} \mathrm{O}_{4}$ with amino groups, the obtained magnetic $\mathrm{Fe}_{3} \mathrm{O}_{4}$ nanoparticle and silane coupling agent $\mathrm{KH}-560$ (named as APTES) were successively dispersed in the mixed solution consist of methyl alcohol and methylbenzene at $110{ }^{\circ} \mathrm{C}$ for $10 \mathrm{~h}$ (Wang et al. 2009). The volume ratio of methyl alcohol to methylbenzene is 1.0 (70 mL:70 mL). Then, the APTES/ $/ \mathrm{Fe}_{3} \mathrm{O}_{4}$ nanoparticle was obtained.

\section{Preparation of GO-coated and GOF-coated} cellulose fabric

The graphene oxide (GO) sheets were synthesized from graphite flakes using a modified Hummers' method, as reported previously (Hummers;Offeman 1958). The weight ratio of graphite, potassium permanganate $\left(\mathrm{KMnO}_{4}\right)$ and sulfuric acid $\left(\mathrm{H}_{2} \mathrm{SO}_{4}\right.$, $98 \%$ ), was 1:3:14. Then the mixture was stirred at $0{ }^{\circ} \mathrm{C}$ for $2 \mathrm{~h}$ and heated to $45^{\circ} \mathrm{C}$ for $30 \mathrm{~min}$. Then the deionized water $(280 \mathrm{~mL})$ and $\mathrm{H}_{2} \mathrm{O}_{2}(10 \mathrm{~mL} 30 \%)$ were added and stirred for $15 \mathrm{~min}$. After the reaction completely static, the supernatant liquid was removed and the residues was washed with hydrochloric acid $(\mathrm{HCl})$ and water, then, centrifuged and washed with water many times to neutral. The GO solution was finally obtained.
To produce the GOF solution, the obtained GO (2 g) was diluted with water $(100 \mathrm{~mL})$ and then, the APTES/ $/ \mathrm{Fe}_{3} \mathrm{O}_{4}$ nanoparticle was added according to the ratio of GO to APTES/ $/ \mathrm{Fe}_{3} \mathrm{O}_{4}$ nanoparticle. The weight ratio of GO and APTES/ $/ \mathrm{Fe}_{3} \mathrm{O}_{4}$ was respectively 95:5, 90:10, 85:15 and 80:20.

GO-coated and GOF-coated fabrics were prepared via the multi-dipping-drying treatment of GO and GOF dispersions on the cellulose fabrics (warp: $25 \mathrm{~cm}$; filling: $2 \mathrm{~cm}$ ), as shown in Fig. 1. Firstly, the GO solution $(250 \mathrm{~mL})$ with mass fraction of $0.61 \%$ was diluted in water. Then, the cellulose fabrics were dipped into the prepared dispersion, sonicated for $30 \mathrm{~min}$ at room temperature, without the external magnetic field and dried at $50{ }^{\circ} \mathrm{C}$ for $12 \mathrm{~h}$. This process was known as the assembly of the GO layers onto cellulose fabrics. According to this method, the GO layers were assembled layer-by-layer onto the surface of cellulose fabrics for five times. In a similar way, for the GOF-coated cellulose fabrics, the layer-by-layer assembly of GOF layers onto the surface of cellulose fabrics was carried out in an external magnetic field.

Preparation of RGO-coated and RGOF-coated cellulose fabrics

RGO-coated cellulose fabrics were prepared by subjecting GO coated cellulose fabrics to a reductive treatment. Based on the number of the repeated times of dipping-drying treatments, the five GO coated fabrics (GO-100/0-1, GO-100/0-2, GO-100/0-3, GO100/0-4 and GO-100/0-5) were respectively immersed in the $\mathrm{HI}$ solutions $(47 \%, 100 \mathrm{~mL})$ and was kept at $80{ }^{\circ} \mathrm{C}$ for 3 min under constant stirring. Then, the fabrics were washed five times with distilled water in order to remove the excessive reducing agents. Finally, the fabrics were dried at $50{ }^{\circ} \mathrm{C}$ and different RGO-100/0 samples were collected (RGO100/0-1, RGO-100/0-2, RGO-100/0-3, RGO-100/ 0-4 and RGO-100/0-5). The different proportions of RGOF-coated cellulose fabrics (RGOF-95/5, RGOF90/10, RGOF-85/15 and RGOF-80/20) were prepared by the similar methods. According to the number of the repeated times of dipping-drying treatments, twenty RGOF-coated cellulose fabrics were obtained. 


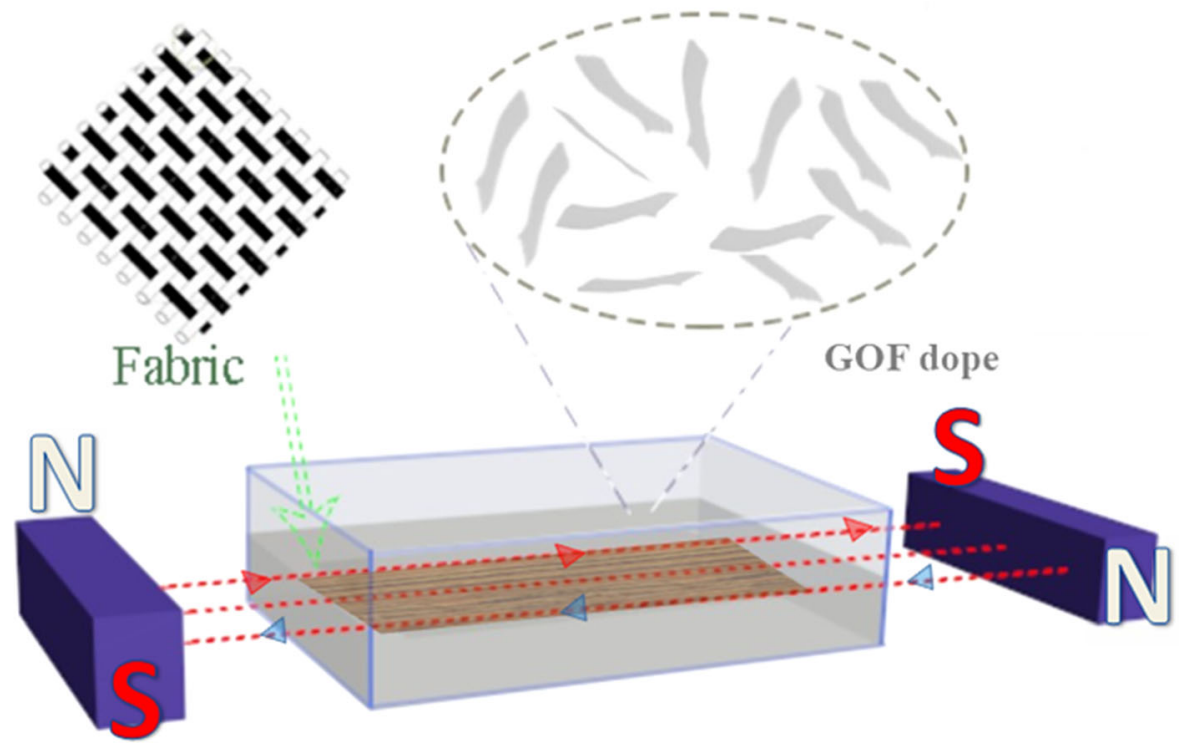

Fig. 1 Schematic diagram of GO-coated cellulose fabrics. The blue box denoted the magnetic iron

\section{Characterization}

In this work, we fabricated flexible conductive fabrics via the layer-by-layer assembly of grapheme oxide (GO) gafted with ferroderric oxide on cellulose fabrics in an external magnetic field and followed by chemical reduction. The structure of the untreated cellulose fabrics, GO-coated fabrics and their reducing products was confirmed via X-ray photoelectron spectroscopy (XPS) with an XSAM800 (Kratos, UK) by using monochromatized $\mathrm{Al} \mathrm{Ka}(1486.6 \mathrm{eV})$ at $180 \mathrm{~W}$ $(15 \mathrm{~mA}, 12 \mathrm{kV})$. The morphology of the different fabrics was examined using field emission scanning electron microscopy (FESEM, JEOL JSM-7100F) at an acceleration voltage $5 \mathrm{kV}$ and transmission electron microscopy (TEM, JEM-2100 Electron Microscope in Japan) using an acceleration voltage of 200 kV. Polarized optical microscopy (POM) measurements of the samples were conducted with a DM2700P Leica Microsystems (German). Moreover, the flexible electrical conductivity of the different fabrics was measured and the relationship between the assembled structure and properties was further investigated. The electrical conductivities of the RGO- and RGOF-coated fabrics were tested according to the International electro technical commission 93-1980 standard (Zhao et al. 2015; Zhang et al. 2016). In detail, two copper electrodes with the spacing of $5 \mathrm{~cm}$ were placed on the specimens (width: $1.5 \mathrm{~cm}$; thickness: $0.036 \mathrm{~cm}$ ) and the electrical resistivity could be calculated by the equation:

$\rho_{v}=R \cdot \frac{S}{L}$

where $R$ is the electrical resistivity of the fabric $(\Omega), \mathrm{L}$ is the distance between the two electrodes $(\mathrm{cm})$ and $S$ is the cross-sectional area of the fabric $\left(\mathrm{cm}^{2}\right)$.

\section{Results and discussion}

The structure and component of different cellulose fabrics

To investigate the chemical changes during the reaction of APTES/Fe ${ }_{3} \mathrm{O}_{4}$ and $\mathrm{GO}$ nanosheets and the reduction of the resulting cellulose fabrics, the XPS measurements were performed and the results are shown in Fig. 2. Figure 2a showed the XPS survey spectra of pure cellulose fabrics (CF), GO-100/0-3, GOF-80/20-3, RGO-100/0-3, RGOF-80/20-3 and these samples were mainly composed of $\mathrm{C}, \mathrm{O}, \mathrm{N}$ and $\mathrm{Fe}$ elements. Figure $2 \mathrm{~b}, \mathrm{c}$ showed the $\mathrm{C} 1 \mathrm{~s}$ peaks of the five samples. For GO-100/0-3 sample, the peak position of C1s was observed at $284.6 \mathrm{eV}, 286.0 \mathrm{eV}$, $287.9 \mathrm{eV}$. Compared to C1s of GO-100/0-3, the peaks at $284.6 \mathrm{eV}, 285.9 \mathrm{eV}, 287.5 \mathrm{eV}, 288.6 \mathrm{eV}$ for GOF$80 / 20-3$ was corresponding to the formation of 

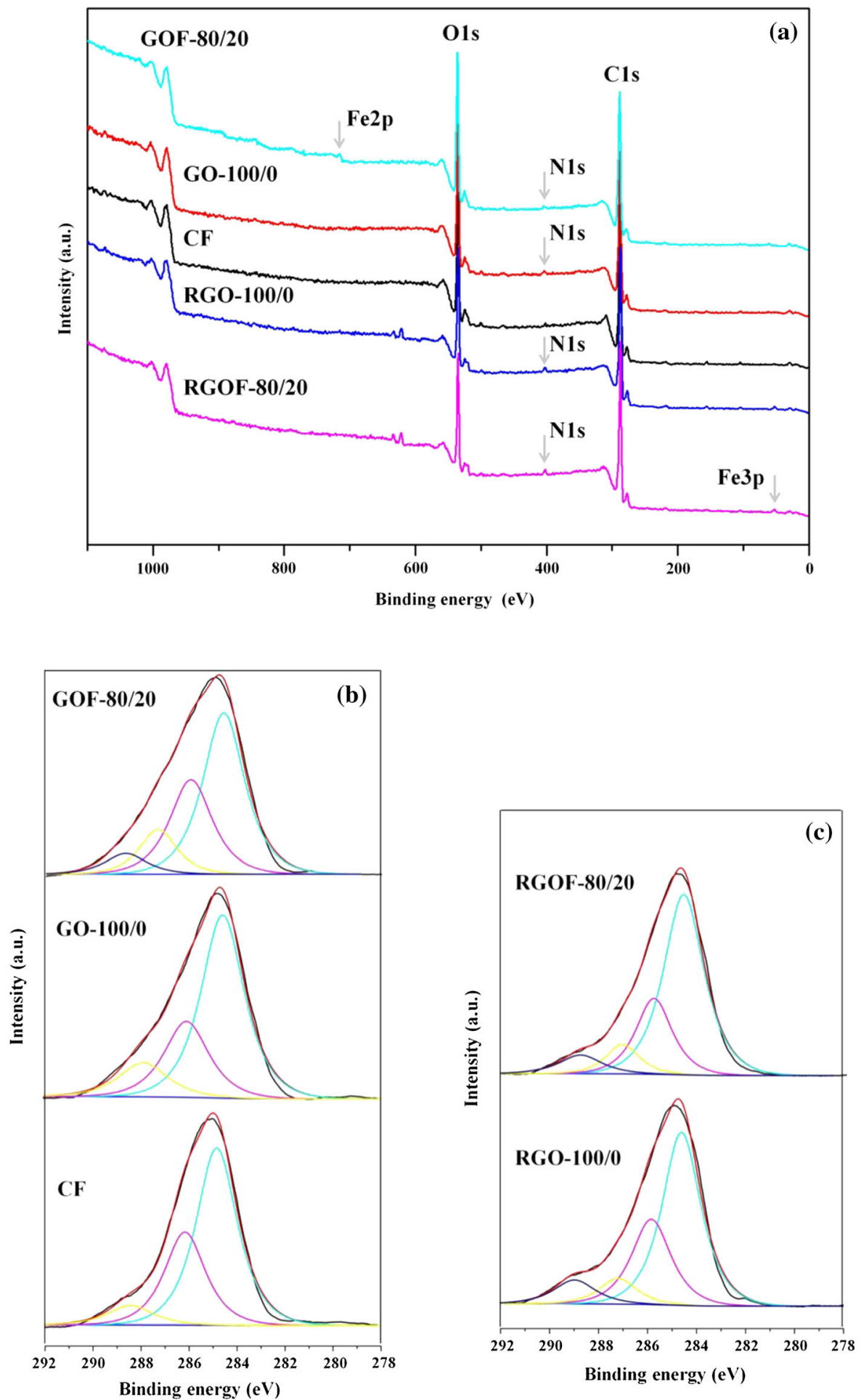

Fig. 2 XPS spectra of the samples. a XPS survey spectra of CF, GO-100/0, GOF-80/20, RGO-100/0, RGOF-80/20 fabrics. b C1s of CF, GO-100/0, GOF-80/20 fabrics. c C1s of RGO-100/0, RGOF-80/20 fabrics 
chemical bonds between APTES/ $/ \mathrm{Fe}_{3} \mathrm{O}_{4}$ nanoparticle and $\mathrm{GO}$, which was agreement with the experimental results (Ramasundaram et al. 2014). It can be seen from Table 1 that fractions of carbon and oxygen in GO-100/0-3 were 74.1 and $24.8 \%$ by calculating the area of the corresponding peak in the deconvoluted $\mathrm{C} 1 \mathrm{~s}$ XPS spectra. After reduction, the $\mathrm{C} / \mathrm{O}$ atomic ratio was significantly increased in RGO-100/0-3 sample (C: $76.7 \%$, O: $21.5 \%$ ), which suggested that the remove of some functional groups and supported our previous results (Zhang et al. 2019). In addition, the iron content after reduction was decreased from $0.5 \%$ in GOF-80/20-3 to 0 in RGOF-80/20-3, which indicated that the iron ion was also reduced. The decreased iron content would have a great influence on the structural change from the magnetic GOF-80/20-3 fabrics to non-magnetic RGOF-80/20-3, which could be demonstrated by our previous results (Zhang; $\mathrm{Wu}$ et al. 2019).

The pure cellulose fabrics and GO-based coated cellulose fabrics through magnetic induced selfassembly

In order to investigate the morphology of RGO- and RGOF-coated fabrics with number of the deposition cycles in an external magnetic field, the evolution of texture of these fabrics were observed by FESEM, as shown in Fig. 3. With the same number of deposition cycles, the pristine RGO coated-fabrics were smoothly covered the entire fabric surface. It is observed that the greater the weight assigned to the APTES/ $/ \mathrm{Fe}_{3} \mathrm{O}_{4}$ nanoparticles; the more increase of surface coarseness will be increased to that coated fabrics. Additionally, the surface coarseness increased with the increasing number of deposition cycles in same mass ratios. To be specific, with the deposition cycles increasing from

Table 1 Elemental content of different samples

\begin{tabular}{lllll}
\hline Samples & C $(\%)$ & O (\%) & N $(\%)$ & Fe (\%) \\
\hline CF & 74.6 & 24.5 & & \\
GO-100/0 & 74.1 & 24.8 & 1.1 & \\
RGO-100/0 & 76.7 & 21.5 & 1.2 & \\
GOF-80/20 & 73.2 & 25.7 & 0.6 & 0.5 \\
RGOF-80/20 & 78.7 & 19.9 & 1.2 & \\
\hline
\end{tabular}

Fig. 3 FESEM images of RGO- and RGOF-coated fabrics with number of the deposition cycles in an external magnetic field. a From left to right, the number was arranged in accordance with deposition cycles from low to high and denoted as one cycle, two cycles, three cycles, four cycles and five cycles with GO and APTES/ $/ \mathrm{Fe}_{3} \mathrm{O}_{4}$ nanoparticles in different mixture ratios. b The magnification of one cycle, three cycles and five cycles shown in a

1 to 3 , it is displayed that the morphologies of RGOF sheets gradually change into the well-aligned alternating ordered structures along the external-field direction. The red dotted box shown in Fig. 3 exhibited the well arrayed coated-fabrics with GO and APTES/ $/ \mathrm{Fe}_{3} \mathrm{O}_{4}$ nanoparticles in different mixture ratios. However, with further increases deposition cycles, i.e., from 4 to 5 times, the multilayer fabrics appeared to be sloped downwards to the right, especially the fabric located at bent locations, which suggested that the higher loading of APTES/ $/ \mathrm{Fe}_{3} \mathrm{O}_{4}$ nanoparticles was unfavorable for the periodic structural rearrangements of RGOF layers.

In order to confirm that the change of macroscopic alignment of RGOF in FESEM could be controlled by applying a magnetic field, polarized optical microscopy (POM) measurements were performed for GOF dispersion liquid (Fig. 4). It can be seen from Fig. 4 that the texture of GOF dispersion liquid was separated by disclinations without magnetic field (see Fig. 4a), however, it was appeared to be directional arrangement and eventually converged to form a large domain (see Fig. 4b) in the present of magnetic field. It was indicated that the macroscopic orientation of GOF dispersion liquid could be tuned by the magnetic field.

The morphological changes of GOF dispersion was monitored by TEM measurements to further understand the magnetic alignment displayed in Fig. 2 after applying an external magnetic field and the results are shown in Fig. 5. In the absence of the magnetic field, as shown in Fig. 5a, the GOF nanosheets exhibited the irregular dispersion morphology with the agglomerates of $\mathrm{APTES} / \mathrm{Fe}_{3} \mathrm{O}_{4}$ nanoparticles, which resulted from the interaction between GOF nanosheets and APTES/ $/ \mathrm{Fe}_{3} \mathrm{O}_{4}$ nanoparticles and the intra-molecular hydrogen bonding between GOF sheets(Chen et al. 2008; Tong et al. 2019). In the presence of a magnetic field, the magnetic force of magnetic field to APTES/ $\mathrm{Fe}_{3} \mathrm{O}_{4}$ nanoparticles would compress the GOF 

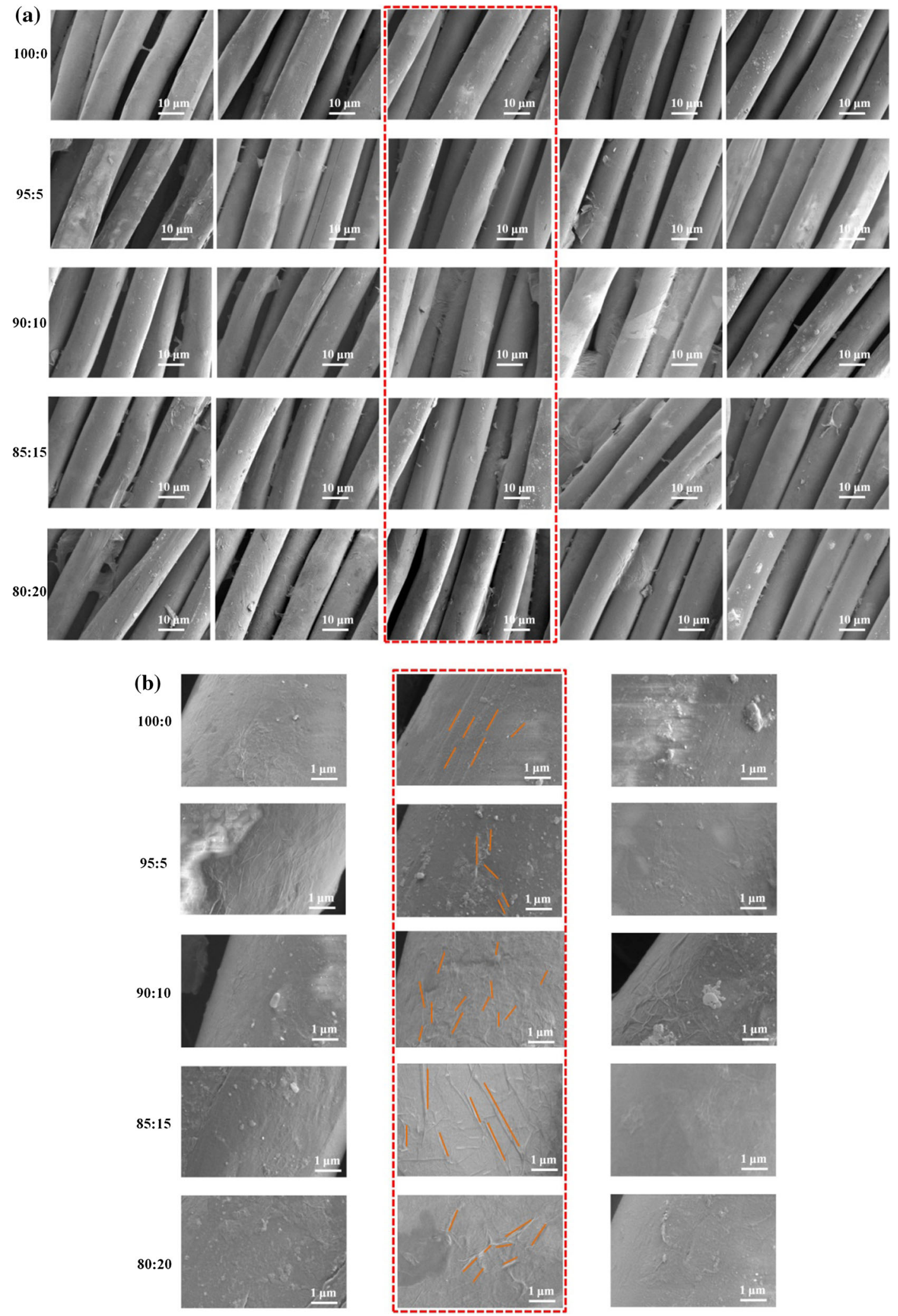
Fig. 4 POM images of GOF-90/10 dispersion liquid with magnetic induction
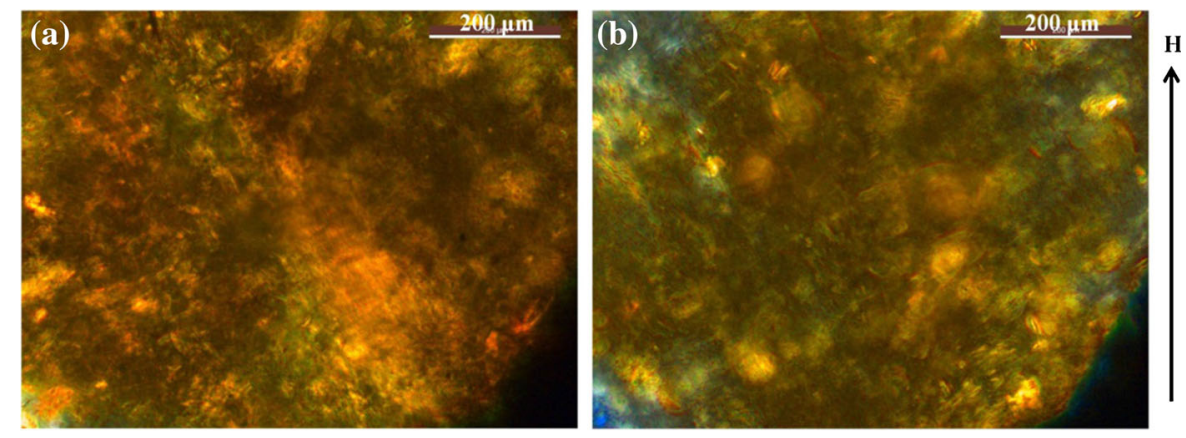
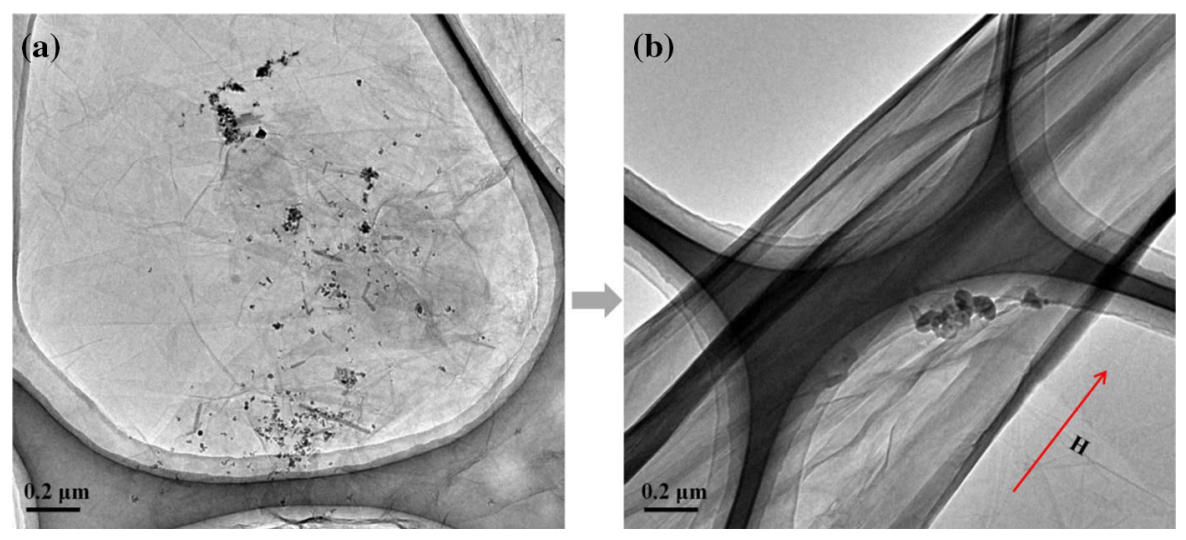

Fig. 5 TEM images of GOF nanosheets without $\mathbf{a}$ and with magnetic induction $\mathbf{b}$

nanosheets into a parallel band along the direction of the magnetic field (Fig. 5(b)), which would contribute to control the microscopic structures of GOF sheets.

The mechanism of magnetic induced selfassembly of GOF-coated cellulose fabrics

According to the self-assembly theory of magneticfiled induction, there are five kinds of dominant interactions in magnetic-field self-assembly system, including the interaction force between external magnetic field and APTES/ $/ \mathrm{Fe}_{3} \mathrm{O}_{4}$ nanoparticles, mutual attraction between magnetic dipole, van der Waals interaction between magnetic APTES/ $\mathrm{Fe}_{3} \mathrm{O}_{4}$ nanoparticles, steric hindrance of APTES/ $/ \mathrm{Fe}_{3} \mathrm{O}_{4}$ nanoparticles, the resistance between APTES/ $/ \mathrm{Fe}_{3} \mathrm{O}_{4}$ nanoparticles during motion (Helgesen et al. 1988; Morimoto;Maekawa 1999; Butter et al. 2003; Lalatonne et al. 2004; Pileni; Ngo 2005). The main driving force generated by self-assembly of GOF coated cellulose fabrics in an external magnetic field are the magnetic force of magnetic field to APTES/Fe ${ }_{3} \mathrm{O}_{4}$ nanoparticles and the interaction between magnetic doublets. To be specific, the magnetic easy axis of APTES/ $/ \mathrm{Fe}_{3} \mathrm{O}_{4}$ nanoparticles with magnetic anisotropy was oriented to the magnetic line of force when the APTES/Fe ${ }_{3} \mathrm{O}_{4}$ nanoparticles in GOF coated cellulose fabrics were magnetized with the magnetic field turned on. The magnetized magnetic APTES/ $/ \mathrm{Fe}_{3} \mathrm{O}_{4}$ nanoparticles become a magnetic dipole and correspondingly, the magnetic dipole moment between the magnetic dipoles could be generated. The mutual repulsion or attraction between the magnetic dipole moments could cause the magnetic APTES/ $/ \mathrm{Fe}_{3} \mathrm{O}_{4}$ nanoparticles to be oriented, which determined the self-assembly behavior of RGOF coated cellulose fabrics (Helgesen, Skjeltorp et al. 1988; Butter, Bomans et al. 2003). The magnetic force acting on the APTES/ $/ \mathrm{Fe}_{3} \mathrm{O}_{4}$ nanoparticles can be written as

$F_{X}=V_{p} \frac{\mu_{0} \chi H}{1+\chi N} \frac{d H}{d x}$

where $V_{p}$ is the volume of APTES/ $/ \mathrm{Fe}_{3} \mathrm{O}_{4}$ nanoparticle; $\mu_{0}$ is the vacuum permeability; $\chi$ is the magnetic 
susceptibility; $N$ is the geometry factor and the value is 0.33 for the spherical particle; $H$ is the magnetic field strength and $x$ is the distance between APTES/ $/ \mathrm{Fe}_{3} \mathrm{O}_{4}$ nanoparticle and magnetic iron. It is noticed from Eq. (2) that the smaller the value for $x$ can change to a larger magnetic force, which would speed up the movement of APTES/ $/ \mathrm{Fe}_{3} \mathrm{O}_{4}$ nanoparticles, leading to greater macroscopic alignment of GOF nanosheets.

Electrical and thermal conductivity of different coated cellulose fabrics

Figure 6 showed the obtained cycles-volume resistive curves of the fabric specimens in warp directions after induction by the magnetic field. With an increase of the deposition cycles from 1 to 3 , the value of different coated fabrics decreased gradually under induction of magnetic field, resulting from the morphologies of RGO and RGOF Nano sheets shown in Fig. 3. Particularly, the value of RGOF-85/15-3 fabrics exhibited a low volume resistivity of $64.8 \Omega \cdot \mathrm{cm}$, it's four times smaller than the Tian's work (Tian et al.

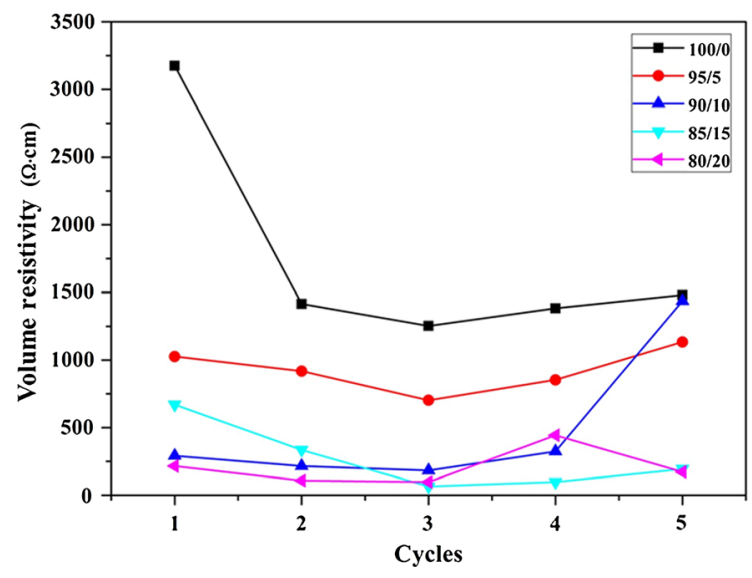

Fig. 6 Electrical conductivity values for different RGO- and RGOF-coated cellulose fabrics

Table 2 Comparison of our study with the reported cotton fabric coating rGO in terms of electrical conductivity
2016) (shown in Table 2) and about 2 times that of pure RGO-coated viscose fabrics of 5-RGO-c-VF with 5 number of deposition cycles $(137.94 \Omega \cdot \mathrm{cm})$ in the previous work (Zhang;Cao et al. 2016). At the same time, the surface resistivity is about the same as the Zhao et al.'s work it handles five times (Zhao; Wang et al. 2020), indicating the increased electrical conductivity of the coated RGOF-85/15-3 fabrics, which revealed that a certain amount of intervention of APTES/ $\mathrm{Fe}_{3} \mathrm{O}_{4}$ nanoparticles under the external magnetic field could improve the mobility of charge carriers effectively among the sheets of fabrics (Shenoy;Reddy et al. 2008). It was also demonstrated that the well-aligned alternating ordered structures of RGOF-85/15-3 fabrics shown in Fig. 3 could improve the electrical conductivity of coated cellulose fabrics. Further, compared with those of coated cellulose fabrics of pure RGO, the results suggested that the magnetic induced coated cellulose fabrics of RGOF sheets grafted with Ferro ferric oxide could not only to retain the high electrical conductivity, but also to decrease the number of the deposition cycles and the graphene oxide consumption. The samples with more coating of RGOF and RGOF showed a slightly increase in volume resistivity due to the increased surface coarseness. Additionally, with the same number of cycles of the layer-by-layer assembly, an increase in the weight ratio of APTES/ $/ \mathrm{Fe}_{3} \mathrm{O}_{4}$ nanoparticles would be beneficial for increasing the electrical conductivity of the coated fabrics.

To further confirm the electrical conductivity and durability of the GO and GOF deposited fabrics, the cyclic resistance change response of the deposited fabrics was tested after being washed with deionized water twelve times and the washing step kept $30 \mathrm{~min}$ in room temperature each time, as shown in Fig. 7. It can be found from Fig. 7 that after twelve times water laundering, the resistance of the RGOF-80/20-3 fabric slightly increased from $0.10 \mathrm{k} \Omega \mathrm{cm}$ (before washing) to $1.31 \mathrm{k} \Omega \mathrm{cm}$ (after washing) compared with that of

\begin{tabular}{lll}
\hline Ref & Volume resistivity $(\Omega \mathrm{cm})$ & Surface resistivity $(\mathrm{k} \Omega / \mathrm{sq})$ \\
\hline Tian; Hu et al. (2016) & 229.0 & - \\
This study & 64.8 & 0.65 \\
Zhang; Cao et al. (2016) & 137.9 & - \\
Zhao; Wang et al. (2020) & - & 0.54 \\
Ren; Wang et al. (2017) & - & 0.90 \\
\hline
\end{tabular}




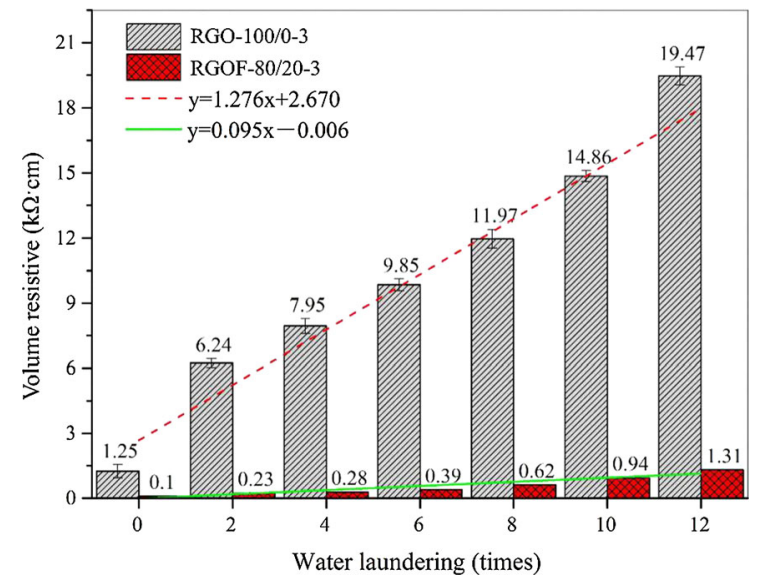

Fig. 7 The change in volume resistive values for RGO- and RGOF-coated cellulose fabrics with the water laundering times from 0 to 12. The number of deposition cycles is 3 times for RGO- and RGOF-coated fabrics

the RGO-100/0-3 fabric (before washing: $1.25 \mathrm{k} \Omega \mathrm{cm}$; after washing: $19.47 \mathrm{k} \Omega \mathrm{cm}$ ), indicating that the degradation of its electrical conductivity performance is not obvious after washing. It was also demonstrated that the coated-fabrics with GO and APTES/Fe ${ }_{3} \mathrm{O}_{4}$ nanoparticles under an external magnetic field possessed excellent water laundering durability.

In order to investigate the change in microstructure to the influence of thermal conductivity property under induction of external magnetic field, the temperature response time of the different RGO- and RGOF-coated fabrics to $55^{\circ} \mathrm{C}$ was observed by the change of label color, shown in Fig. 8, Table 3 and Movie S1-5. The minimal response time was respectively $4.998 \mathrm{~s}$, $4.898 \mathrm{~s}, 4.781 \mathrm{~s}, 4.374 \mathrm{~s}$ and $3.963 \mathrm{~s}$ in different weight ratios of RGO-100/0, RGOF-95/5, RGOF-90/ 10, RGOF-85/15 and RGOF-80/20 when the deposition cycle was 3 . It was demonstrated that the wellaligned alternating ordered structures shown in the red dotted box of Fig. 3 could improve the thermal conductivity of coated cellulose fabrics.

\section{Conclusions}

In summary, we fabricated the cellulose fabrics via a layer-by-layer magnetic-field-induced self-assembly of graphene oxide sheets grafted with ferroferric oxide (GOF) and followed by chemical reduction. The FESEM results revealed that the morphologies of RGOF sheets in same APTES/ $/ \mathrm{Fe}_{3} \mathrm{O}_{4}$ nanoparticles mass ratios gradually formed the well-aligned alternating ordered structures along the external-field direction when the deposition cycles increased from 1 to 3 , however, the higher loading of APTES/ $/ \mathrm{Fe}_{3} \mathrm{O}_{4}$ nanoparticles was unfavorable for the periodic structural rearrangements of RGOF layers. It resulted from

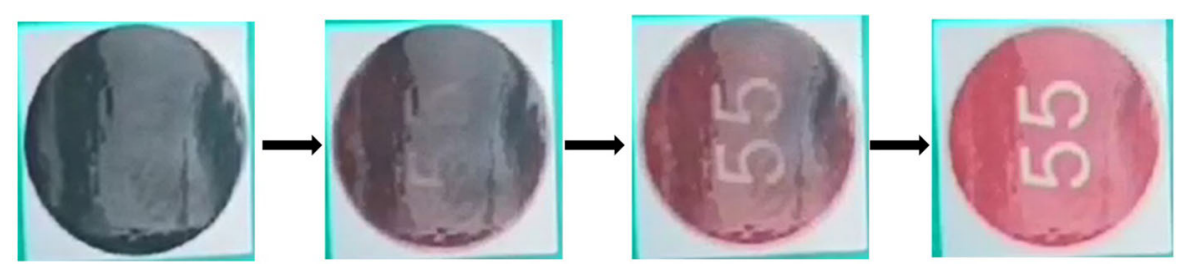

Fig. 8 Schematic diagram of temperature response of the coated fabric of RGO-100/0-5

Table 3 Temperature response time of the coated fabrics

\begin{tabular}{llllll}
\hline Depositioncycles & \multicolumn{5}{l}{ Response time $(\mathrm{s})$} \\
\cline { 2 - 6 } & RGO-100/0 & RGOF-95/5 & RGOF-90/10 & RGOF-85/15 & RGOF-80/20 \\
\hline 1 & 5.165 & 4.446 & 4.975 & 4.821 & 4.507 \\
2 & 5.031 & 5.008 & 4.963 & 4.628 & 4.251 \\
3 & 4.998 & 4.898 & 4.781 & 4.374 & 3.963 \\
4 & 5.249 & 5.036 & 5.002 & 4.630 & 4.852 \\
5 & 5.631 & 5.577 & 5.487 & 5.217 & 5.092 \\
\hline
\end{tabular}


the synergistic interactions of magnetic induction force on magnetic doublet, hydrogen bonds and van der Waals. Due to the presence of such ordered structures, the value of RGOF-85/15-3 fabrics exhibited a low volume resistivity of $64.8 \Omega \cdot \mathrm{cm}$ compared with that of pure RGO-coated viscose fabrics of 5-RGO-c-VF (137.94 $\Omega \mathrm{cm}$ ) in the previous work, which revealed that the magnetic induced coated cellulose fabrics of RGOF sheets grafted with ferroferric oxide could not only to retain the high electrical conductivity, but also to decrease the number of the deposition cycles and the graphene oxide consumption. Moreover, after twelve times water laundering, the resistance of the RGOF-80/20-3 fabric slightly increased by $1.21 \mathrm{k} \Omega^{\mathrm{r}} \mathrm{cm}$ compared with that of the RGO-100/0-3 fabric $\left(18.22 \mathrm{k} \Omega^{`} \mathrm{~cm}\right)$, indicating that the GOF coated-fabrics under an external magnetic field possessed excellent water laundering durability. In addition, the results of thermosensitivity measurement was demonstrated that the well-aligned alternating ordered structures could improve the thermal conductivity of coated cellulose fabrics. Therefore, this work would provide fundamental insight into the relationship between the different structure of the assembly layer-deposited fabrics and their properties, which shed light on the design of next-generation wearable electronics, strain sensors, smart actuators and bioelectrodes and so on.

Acknowledgments We gratefully acknowledge the financial support from the National Natural Science Foundation of China (61704068), the Science and Technology Innovation Project for Undergraduates in Zhejiang Province (2019R417015), China Scholarship Council fund (201908330045) , "Baiqing" Training Plan of Jiaxing University (CD70619036) and the Scientific Research Allowance of Dezhou University (2019xjrc336).

Open Access This article is licensed under a Creative Commons Attribution 4.0 International License, which permits use, sharing, adaptation, distribution and reproduction in any medium or format, as long as you give appropriate credit to the original author(s) and the source, provide a link to the Creative Commons licence, and indicate if changes were made. The images or other third party material in this article are included in the article's Creative Commons licence, unless indicated otherwise in a credit line to the material. If material is not included in the article's Creative Commons licence and your intended use is not permitted by statutory regulation or exceeds the permitted use, you will need to obtain permission directly from the copyright holder. To view a copy of this licence, visit http://creativecommons.org/licenses/by/4.0/.

\section{References}

Bao L, Zang J, Li X (2011) Flexible Zn2SnO4/MnO2 Core/shell nanocable-carbon microfiber hybrid composites for highperformance supercapacitor electrodes. Nano Lett 11:1215-1220

Butter K, Bomans PHH, Frederik PM, Vroege GJ, Philipse AP (2003) Direct observation of dipolar chains in iron ferrofluids by cryogenic electron microscopy. Nature Mater 2:88-91

Chen H, Müller MB, Gilmore KJ, Wallace GG, Li D (2008) Mechanically strong, electrically conductive and biocompatible graphene paper. Adv Mater 20:3557-3561

Chen Y, Guo F, Jachak A, Kim S-P, Datta D, Liu J, Kulaots I, Vaslet C, Jang HD, Huang J, Kane A, Shenoy VB, Hurt RH (2012) Aerosol synthesis of cargo-filled graphene nanosacks. Nano Lett 12:1996-2002

Gao Z, Schwab Y, Zhang Y, Song N, Li X (2018) Ferromagnetic nanoparticle-assisted polysulfide trapping for enhanced lithium-sulfur batteries. Adv Funct Mater 28(20): 1800563

Gómez-Navarro C, Burghard M, Kern K (2008) Elastic properties of chemically derived single graphene sheets. Nano Lett 8:2045-2049

Grancarić AM, Jerković I, Koncar V, Cochrane C, Kelly FM, Soulat D, Legrand X (2017) Conductive polymers for smart textile applications. J Ind Text 48:612-642

Gu JF, Gorgutsa S, Skorobogatiy M (2010) Soft capacitor fibers using conductive polymers for electronic textiles. Smart Mater Struct 19:115006

Han TH, Kim J, Park JS, Park CB, Ihee H, Kim SO (2007) Liquid crystalline peptide nanowires. Adv Mater 19:3924-3927

Hao W, Chiou K, Qiao Y, Liu Y, Song C, Deng T, Huang J (2018) Crumpled graphene ball-based broadband solar absorbers. Nanoscale 10:6306-6312

Heidari M, Bahrami SH, Ranjbar-Mohammadi M, Milan PB (2019) Smart electrospun nanofibers containing PCL/gelatin/graphene oxide for application in nerve tissue engineering. Mater Sci Eng, C 103:109768

Helgesen G, Skjeltorp AT, Mors PM, Botet R, Jullien R (1988) Aggregation of magnetic microspheres: experiments and simulations. Phys Rev Lett 61:1736-1739

Hu X, Tian M, Xu T, Sun X, Sun B, Sun C, Liu X, Zhang X, Qu L (2020) Multiscale disordered porous fibers for selfsensing and self-cooling integrated smart sportswear. ACS Nano 14:559-567

Huang B, Liu M, Su N, Wu J, Duan W, Gu B-1, Liu F (2009) Quantum manifestations of graphene edge stress and edge instability: a first-principles study. Phys Rev Lett 102:166404

Hummers WS, Offeman RE (1958) Preparation of graphitic oxide. J Am Chem Soc 80:1339-1339

Kim JE, Han TH, Lee SH, Kim JY, Ahn CW, Yun JM, Kim SO (2011a) Graphene oxide liquid crystals. Angew Chem 123:3099-3103

Kim TY, Kwon SW, Park SJ, Yoon DH, Suh KS, Yang WS (2011b) Self-organized graphene patterns. Adv Mater 23:2734-2738 
Lalatonne Y, Richardi J, Pileni MP (2004) Van der Waals versus dipolar forces controlling mesoscopic organizations of magnetic nanocrystals. Nat Mater 3:121-125

Morimoto H, Maekawa T (1999) Dynamic analysis of a ferromagnetic colloidal system. Int $\mathbf{J}$ Mod Phys $B$ 13:2085-2092

Pileni M-P, Ngo A-T (2005) Mesoscopic structures of maghemite nanocrystals: fabrication, magnetic properties and uses. ChemPhysChem 6:1027-1034

Ramadoss A, Saravanakumar B, Kim SJ (2015) Thermally reduced graphene oxide-coated fabrics for flexible supercapacitors and self-powered systems. Nano Energy 15:587-597

Ramasundaram S, Jung JH, Chung E, Maeng SK, Lee SH, Song KG, Hong SW (2014) Increasing hydrophobicity of poly(propylene) fibers by coating reduced graphene oxide and their application as depth filter media. Carbon 70:179-189

Ren J, Wang C, Zhang X, Carey T, Chen K, Yin Y, Torrisi F (2017) Environmentally-friendly conductive cotton fabric as flexible strain sensor based on hot press reduced graphene oxide. Carbon 111:622-630

Shenoy VB, Reddy CD, Ramasubramaniam A, Zhang YW (2008) Edge-stress-induced warping of graphene sheets and nanoribbons. Phys Rev Lett 101:245501

Shenoy VB, Reddy CD, Zhang Y-W (2010) Spontaneous curling of graphene sheets with reconstructed edges. ACS Nano 4:4840-4844

Song Y, Yang H, Wang Y, Chen S, Li D, Zhang S, Zhang X (2013) Controlling the assembly of graphene oxide by an electrolyte-assisted approach. Nanoscale 5:6458-6463

Sun F, Tian M, Sun X, Xu T, Liu X, Zhu S, Zhang X, Qu L (2019) Stretchable conductive fibers of ultrahigh tensile strain and stable conductance enabled by a worm-shaped graphene microlayer. Nano Lett 19:6592-6599

Tao X, Dong L, Wang X, Zhang W, Nelson BJ, Li X (2010) B4C-nanowires/carbon-microfiber hybrid structures and composites from cotton T-shirts. Adv Mater 22:2055-2059

Tian M, Hu X, Qu L, Zhu S, Sun Y, Han G (2016) Versatile and ductile cotton fabric achieved via layer-by-layer self- assembly by consecutive adsorption of graphene doped PEDOT: PSS and chitosan. Carbon 96:1166-1174

Tong M, Cao J, Chen X, Zhang H, Wu W, Ma H (2019) Selfassembly of chemically modified graphene sheets in an external magnetic field. RSC Adv 9:19457-19464

Wang X, Wang L, He X, Zhang Y, Chen L (2009) A molecularly imprinted polymer-coated nanocomposite of magnetic nanoparticles for estrone recognition. Talanta 78:327-332

Xu Z, Sun H, Zhao X, Gao C (2013) Ultrastrong fibers assembled from giant graphene oxide sheets. Adv Mater 25:188-193

Zeng M, Wang L, Liu J, Zhang T, Xue H, Xiao Y, Qin Z, Fu L (2016) Self-Assembly of graphene single crystals with uniform size and orientation: the first $2 \mathrm{~d}$ super-ordered structure. J Am Chem Soc 138:7812-7815

Zhang H, Cao J, Wu W, Cao Z, Ma H (2016) Layer-by-layer assembly of graphene oxide on viscose fibers for the fabrication of flexible conductive devices. Cellulose 23:3761-3770

Zhang H, Wu W, Cao J, Tong M, Xu H, Mao Z, Ma H (2019) Magnetic induced wet-spinning of graphene oxide sheets grafted with ferroferric oxide and the ultra-strain and elasticity of sensing fiber. Compos Part B-Eng 170:1-10

Zhao C, Shu K, Wang C, Gambhir S, Wallace GG (2015) Reduced graphene oxide and polypyrrole/reduced graphene oxide composite coated stretchable fabric electrodes for supercapacitor application. Electrochim Acta 172:12-19

Zhao H, Hu R, Li P, Gao A, Sun X, Zhang X, Qi X, Fan Q, Liu Y, Liu X, Tian M, Tao G, Qu L (2020a) Soft bimorph actuator with real-time multiplex motion perception. Nano Energy 76:104926

Zhao Y, Wang J, Li Z, Zhang X, Tian M, Zhang X, Liu X, Qu L, Zhu S (2020b) Washable, durable and flame retardant conductive textiles based on reduced graphene oxide modification. Cellulose 27:1763-1771

Publisher's Note Springer Nature remains neutral with regard to jurisdictional claims in published maps and institutional affiliations. 